\title{
Mixed-Reality Learning Environments: Integrating Mobile Interfaces with Laboratory Test-beds
}

\begin{abstract}
Even as mobile devices have become increasingly powerful and popular among learners and instructors alike, research involving their comprehensive integration into educational laboratory activities remains largely unexplored. ${ }^{1.1}$ This paper discusses efforts to integrate vision-based measurement and control, augmented reality (AR), and multi-touch interaction on mobile devices in the development of Mixed-Reality Learning Environments $\left(\mathrm{MRLE}^{1}\right)$ that enhance interactions with laboratory test-beds for science and engineering education. A learner points her device at a laboratory test-bed fitted with visual markers while a mobile application supplies a live view of the experiment augmented with interactive media that aid in the visualization of concepts and promote learner engagement. As the learner manipulates the augmented media, her gestures are mapped to commands that alter the behavior of the test-bed on the fly. Running in the background of the mobile application are algorithms performing vision-based estimation and wireless control of the test-bed. In this way, the sensing, storage, computation, and communication $\left(\mathrm{SSCC}^{2}\right)$ capabilities of mobile devices are leveraged to relieve the need for laboratory-grade equipment, improving the cost-effectiveness and portability of platforms to conduct hands-on laboratories. ${ }^{1.1}$ We hypothesize that students using the MRLE platform demonstrate improvement in their knowledge of dynamic systems and control concepts and have generally favorable experiences using the platform. To validate the hypotheses concerning the educational effectiveness and user experience of the MRLEs, an evaluation was conducted with two classes of undergraduate students using an illustrative platform incorporating a tablet computer and motor test-bed to teach concepts of dynamic systems and control. Results of the evaluation study validate the hypotheses. ${ }^{2.2}$ The benefits and drawbacks of the MRLEs observed throughout the study are discussed with respect to the traditional hands-on, virtual, and remote laboratory formats. ${ }^{1.1}$
\end{abstract}

Keywords: applications in subject areas, architectures for educational technology system, improving classroom teaching, interactive learning environments, virtual reality.

\footnotetext{
${ }^{1}$ MRLE: Mixed-Reality Learning Environment

${ }^{2}$ SSCC: Sensing, Storage, Computation, and Communication
} 


\section{Introduction}

The role that laboratories play in providing valuable learning experiences is well-established (Feisel \& Rosa, 2005). In addition to reinforcing concepts introduced by conventional instruction, hands-on laboratory work has been particularly effective at promoting design, collaboration, and social communication skills, which are as desirable in graduates as is a rigorous theoretical background. Although they have enabled learners to put theory into practice, laboratories constantly seek to deliver the most engaging and cost-effective educational experiences possible using the prevailing technology. ${ }^{1.1}$ This is because laboratories require sophisticated laboratory-grade hardware and software to allow for effective interaction with test-beds and visualization of abstract concepts, such as the effects that certain parameters have on the behavior of a system. Thus, limited funding can often reduce access to facilities and inhibit the quality of in-lab activities. Moreover, learners must often focus efforts on installing, calibrating, and troubleshooting equipment at the expense of experimenting and building deep conceptual understanding.

We are living at a time when laptops and desktops are being replaced by smartphones and tablets as the primary personal computers (Bonnington, 2015; Gillett, 2012). In such a rapidly evolving technological landscape, the equipment used in laboratory education is no longer up to date with the expectations of learners, who have become accustomed to mobile, high quality experiences with interactive media. To meet this challenge, virtual and remote laboratories have been made available on mobile devices, allowing learners to interact with simulated or real experiments from anywhere and at any time (Maiti \& Tripathy, 2012). In addition to accessibility and mobility, researchers believe that implementations of mobile remote experiments will better engage and motivate learners (da Silva, Rochadel, Marcelino, Gruber, \& Bilessimo, 2013). However, the mobile-based access to virtual and remote laboratories does not address the challenges facing traditional hands-on laboratories, where there have been few efforts for the comprehensive integration of mobile devices and where research into their potential educational and operational benefits remains largely unexplored. ${ }^{1.1}$

In this paper, a new laboratory approach is presented wherein mobile devices such as smartphones and tablets are employed in roles that go beyond those of traditional graphical interfaces; rather, the devices become responsible for aspects of measurement, estimation, and control of laboratory test-beds. By integrating vision-based control, AR, and touchscreen interaction, mobile devices can provide enhanced interactive experiences with laboratory equipment that may deepen con- 
ceptual understanding and improve learner engagement. Specifically, when the camera of a mobile device is pointed towards a laboratory test-bed, the device augments live video with graphics that learners can directly manipulate to control test-beds and perform experiments. Thus, an intimate connection is made between the digital media running on the interface and the physical dynamics of the test-bed. The term mixed-reality learning environment (MRLE) has been used to refer to such systems (Chang, Lee, Wang, \& Chen, 2010). To demonstrate the proposed approach, the development of an MRLE is presented that uses a mobile interface and a motor test-bed to teach concepts of dynamic systems and control (i.e., damping, stability, and the effects of pole locations on system response). To assist learners' understanding of these concepts, the mobile interface captures a live video of the test-bed with its camera and projects a 3D virtual motor arm that appears attached with the test-bed, providing a visual aid that learners can use to observe phenomena. Moreover, learners intuitively command the angular position of the motor arm by tapping and dragging on the touchscreen to manipulate the virtual arm. Additional interactive media, such as real-time plots of the system response and an interactive pole-zero plot, engage students by allowing them to conduct inquiry-based investigations with the motor test-bed from their personal devices. For example, by tapping on the interactive pole-zero plot, the learner relocates the closed-loop poles of the system, altering the system's dynamics so that the learner can examine the resulting effects on the system response.

The novelty of the work presented in this paper lies both in the technological development and in the research conducted. Specifically, a novel approach is proposed for integrating the capabilities of mobile devices with those of laboratory test-beds in the development of learning environments for teaching dynamic systems and control concepts. In addition to providing interactive mixed-reality visualizations that enhance student engagement, these learning environments reduce the need for significant amounts of laboratory-grade equipment, thus improving the cost-effectiveness of the traditional laboratory. In some cases, these benefits provided by the mobile devices may even facilitate the development of more portable laboratory platforms that utilize learners' devices in various aspects of their operation. ${ }^{1.1}$ To validate the proposed MRLE approach in terms of its educational effectiveness and associated user experiences, a user study was conducted using the developed platform with two classes of undergraduate students. The objective of the study was to examine whether student participants demonstrate significant improvement in content knowledge or report having significantly beneficial experiences after using the MRLE platform compared to before using the MRLE platform and compared to student participants exposed to the content using 
traditional classroom and hands-on laboratory techniques..$^{2.2}$

The paper is organized as follows. In Section 2, we present a review of prior applications that utilize mobile devices as tools in laboratory education. Next, Section 3 provides a detailed overview of the proposed approach, outlines the development of an illustrative platform, and describes the design of evaluation conducted with undergraduate students. Section 4 presents the results of evaluation. Then, Section 5 discusses some observations and rationale for the technical approach and evaluation methodology as well as the evaluation results validating the hypotheses concerning the educational impact and user experience associated with the proposed MRLE approach. Finally, Section 6 offers some concluding remarks and future directions of this work..$^{2.1}$

\section{Background ${ }^{2.1}$}

For many educational institutions, limited resources have made maintaining up-to-date laboratory facilities a serious burden. Moreover, in many areas of the world where there is lack of infrastructure, educators, and finances, access to quality education is simply not available. These issues have attracted research into providing novel technological solutions for science and engineering education that leverage the ubiquitous presence of mobile devices in people's lives. In particular, applications have been developed to leverage students' personal devices into assistive tools for working in science and engineering laboratories (Williams \& Pence, 2011; Nguyen, Barton, \& Nguyen, 2015). These applications, which often can be downloaded at little to no cost, have helped to reduce environmental impact, improve efficiency, increase productivity, improve data quality, and provide immediate access to data (Hesser \& Schwartz, 2013).

Some of the most useful educational applications have been those that provide elegant and interactive visualizations to promote conceptual understanding of abstract concepts. Both virtual and augmented reality techniques have been investigated to provide visually engaging learning experiences, proving especially useful in teaching concepts that rely on spatial reasoning. Examples of applications include those for teaching concepts in primary school science (Kerawalla, Luckin, Seljeflot, \& Woolard, 2006) as well as those for teaching college-level science and engineering concepts (Kaufmann \& Schmalstieg, 2003; Martín-Gutíerrez, Contero, \& Alcañiz, 2010; Maier, Tönnis, \& Klinker, 2009; Liarokapis, Mourkoussis, White, Darcy, Sifniotis, Petridis, et al., 2004). To promote the use of portable AR without the need for hardware such as projected or head-mounted displays, implementations have been examined on mobile devices to teach subjects such 
as astronomy (Tian, Endo, Urata, Mouri, \& Yasuda, 2014). Combining the ability to display visualizations of concepts and data with the computing capacity of mobile devices has allowed for the development of virtual laboratories that run natively on devices without requiring an internet connection or remote cloud-based service. A series of these standalone applications that perform digital signal processing simulations has been developed to teach concepts such as convolution, Fourier analysis, and filter design to engineering students (Liu, Hu, Thiagarajan, Zhang, Ranganath, Banavar, et al., 2012; Ranganath, Thiagarajan, Ramamurthy, $\mathrm{Hu}$, Banavar, \& Spanias, 2012). These authors have acknowledged the significance of user interfaces, including the design of multi-touch gestures, graphical block-based programming, and plots on their ability to enable learners in visualizing algorithms and concepts and to stimulate their interest in performing activities.

The diverse sets of sensors embedded in modern mobile devices have led to their use as portable measurement systems and have impacted areas such as healthcare, transportation, and environmental monitoring (Lane, Miluzzo, Lu, Peebles, Choudhury, \& Campbell, 2010). This sensing capability has led to the use of smartphones and tablets as experimental tools that can aid learners in performing authentic hands-on explorations of physics concepts (Kuhn \& Vogt, 2013). Applications are developed that analyze data recorded by the cameras and motion sensors of learners' personal devices to study phenomena in acoustics, optics, radiation, projectile motion, and centripetal acceleration (Kuhn, Molz, Gröber, \& Frübis, 2014; Klein, Hirth, Gröber, Kuhn, \& Müller, 2014; Klein, Gröber, Kuhn, \& Müller, 2014; Vogt \& Kuhn, 2013). These studies demonstrate that the collection of real, sufficiently accurate data with mobile devices can simplify the experimental setup and allow learners to use their devices to take scientific measurement and experimentation outside of the classroom.

To allow learners to connect to laboratory experiments from their mobile devices, significant research has explored the system architectures (Frank \& Kapila, 2014), software platforms (Maiti \& Tripathy, 2012), and interface design strategies (Orduña, García-Zubia, Irurzun, López-de-Ipiña, \& Rodriguez-Gil, 2011) necessary for developing systems for mobile remote experimentation. With mobile access to laboratory equipment, distant learners can interact with real data from truly anywhere at any time (da Silva et al., 2013), including in creative and spontaneous moments and as part of collaborations with other learners (May, Terkowsky, Haertel, \& Pleul, 2012), availing themselves of investigative experiences that they may not otherwise be able to obtain (de Lima, Rochadel, Silva, Simão, da Silva \& Alves, 2014). However, since current implementations simply adapt the original desktop interfaces for remote laboratories onto smaller screens 
(Orduña et al., 2011) and lack support for the most powerful and attractive mobile technologies (Maiti \& Tripathy, 2012), they fail to address the challenges commonly encountered in traditional hands-on laboratories, such as their requirement for sophisticated and often expensive laboratory-grade hardware and software to operate and interact with laboratory experiments. ${ }^{2.4}$ Thus, an approach that integrates the interactive visualizations provided by virtual and remote laboratories to provide more cost-effective and engaging traditional hands-on laboratories could be a step towards closing the gap between traditional and non-traditional approaches to laboratory education. ${ }^{2.5}$

\section{Methodology 2.1}

The applications discussed in Section 2 demonstrate how the SSCC capabilities of mobile devices have been leveraged to provide learners with the information, tools, data, visualizations, measurements, or remote access needed for effective laboratory learning. For some applications, the SSCC capabilities of devices can be integrated further to provide learners with immersive mixed-reality experiences that can enhance their interactions with test-beds and reduce the need for traditional laboratory-grade hardware and software. In this section, we provide a detailed overview of the proposed approach, followed by an outline of the development of an illustrative platform implementing the approach and a description of the evaluation conducted with undergraduate students.

\subsection{Approach}

Digital cameras and touchscreens are two powerful and attractive components of mobile devices that can be integrated to develop highly visual and interactive interfaces for enhancing laboratory education. In the proposed approach, a learner points a mobile device towards a planar laboratory test-bed from an arbitrary perspective so that the camera can capture video of the test-bed and a mobile application can detect visual markers attached in the plane of the test-bed. By matching the $2 \mathrm{D}$ image locations of the markers to a $3 \mathrm{D}$ model, the application computes the relative pose between the image plane of the device camera and the plane of the test-bed (Frank, Brill, \& Kapila, 2016). With the relative pose computed, positions and orientations of the test-bed's physical elements can be accurately measured in real-world units with respect to the coordinate frame established by the model. These measurements are not only used to render realistic AR elements on the device screen but also in the feedback control of the system itself. Moreover, as learners manipulate the elements on the screen, their gestures are mapped 
to commands for driving the corresponding physical elements of the test-bed and for adjusting parameter values that alter the system's behavior in real time.

As a mobile device is pointed at the test-bed, video frames are captured by the rear-facing camera of the device and processed by the mobile application to detect visual markers attached to critical components of the test-bed. To use the markers' image locations to make accurate physical measurements of the system in real-world coordinates, the pose of its image plane is estimated with respect to a coordinate frame attached to the test-bed. The pose estimation may be performed using several different single-view or multiple-view geometrical techniques (Hartley \& Zisserman, 2003). For the purposes of this study, four markers are arranged in a planar pattern relative to a coordinate frame as specified by a $3 \mathrm{D}$ model. A perspective $n$-point problem is then solved from a set of four 2D-3D point correspondences (Haralick, Joo, Lee, Zhuang, Vaidya, \& Kim, 1989). Although this approach is limited to test-beds whose behavior can be described by planar models, a wide range of platforms in automatic control laboratories meet this constraint, such as servomotors, inverted pendulums, ball and beam test-beds, and planar mechanisms (Apkarian, 1995; Frank \& Kapila, 2016; Frank et al., 2016; Frank, Gómez, \& Kapila, 2015).

Once the relative pose is known between the image plane and the plane of the test-bed, it can be used as a perspective transformation for $(i)$ extraction of accurate physical measurements in real-world coordinates from spatial relations in image coordinates, (ii) realistic projection of virtual elements into the scene, and (iii) mapping of touchscreen gestures performed by the learner to accurate commands for the test-bed. These three functions are integrated by the interface to form a mixed-reality environment that blends the operational space of the test-bed with the visual space of the learner and augments it with interactive virtual graphics for easy monitoring and natural operation of the system. By using this mixed-reality approach to give learners intuitive control over the behavior of the test-bed, the interface becomes transparent, allowing learners with little to no training to immediately have enjoyable and deeply engaging learning experiences.

\subsection{Implementation 2.1}

To illustrate how the proposed MRLE approach may be implemented in practice, the development of a platform is presented that is based on a motor test-bed and used to teach concepts of dynamic systems and control (i.e., stability, damping, and pole-placement controller design). The motor, which has a 6-in. (0.15$\mathrm{m}$.) long rectangular metal arm, is driven by an amplifier that receives control signals from a microcontroller via a digital-to-analog converter (see Figure 1). 


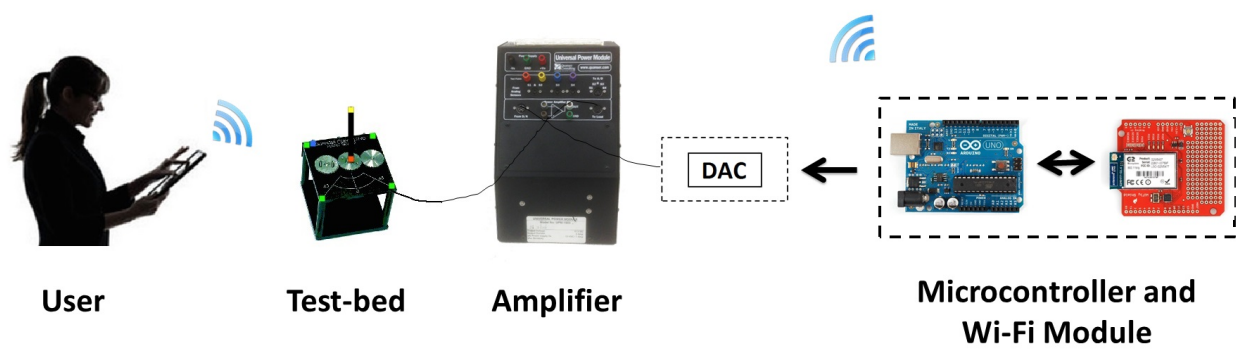

Figure 1: Schematic of the proposed MRLE to teach concepts of dynamic systems and control.

The microcontroller relays to the amplifier control signals that it receives over Wi-Fi from a mobile application executing on a tablet held by the learner. Colored markers affixed to the test-bed are visually detected by the application and used to establish the relative pose between the device and the test-bed and obtain accurate vision-based measurements of the angular position of the motor arm. The measured position is fed into recursive state estimation and feedback control algorithms running on the mobile application to compute the control action, which is wirelessly sent to the microcontroller. The mobile device used in this study is an Apple iPad 2, which has a 9.7-in. (250-mm.), 1024×768 pixel multitouch display, $1 \mathrm{GHz}$ dual-core processor, and a 0.7 -megapixel rear-facing camera. Apple software development supports the use of open source and third-party libraries, such as Open Source Computer Vision Library, Open Source Graphics Library, and CocoaAsyncSocket for image processing, rendering of AR content, and TCP/IP communication, respectively.

The user interface developed for this work (see Figure 2) is split into three main views. In a large view on the right, a live video is shown from the rearfacing camera at 30 frames per second. Projected onto the view is a purple virtual arm that lies in the plane with the actual motor arm in the video and represents the set point for the system. One end of the virtual arm is fixed to the orange marker attached to the corresponding end of the actual motor arm, and the other end pivots about this point - in the same manner that the actual motor arm rotates about its axis - to face locations on the screen that are tapped by the learner.

Three large buttons are provided at the bottom of the view. The first two buttons allow the learner to switch between three modes of operation: connect mode, learn mode, and design mode. Each of these three modes change the display in the 


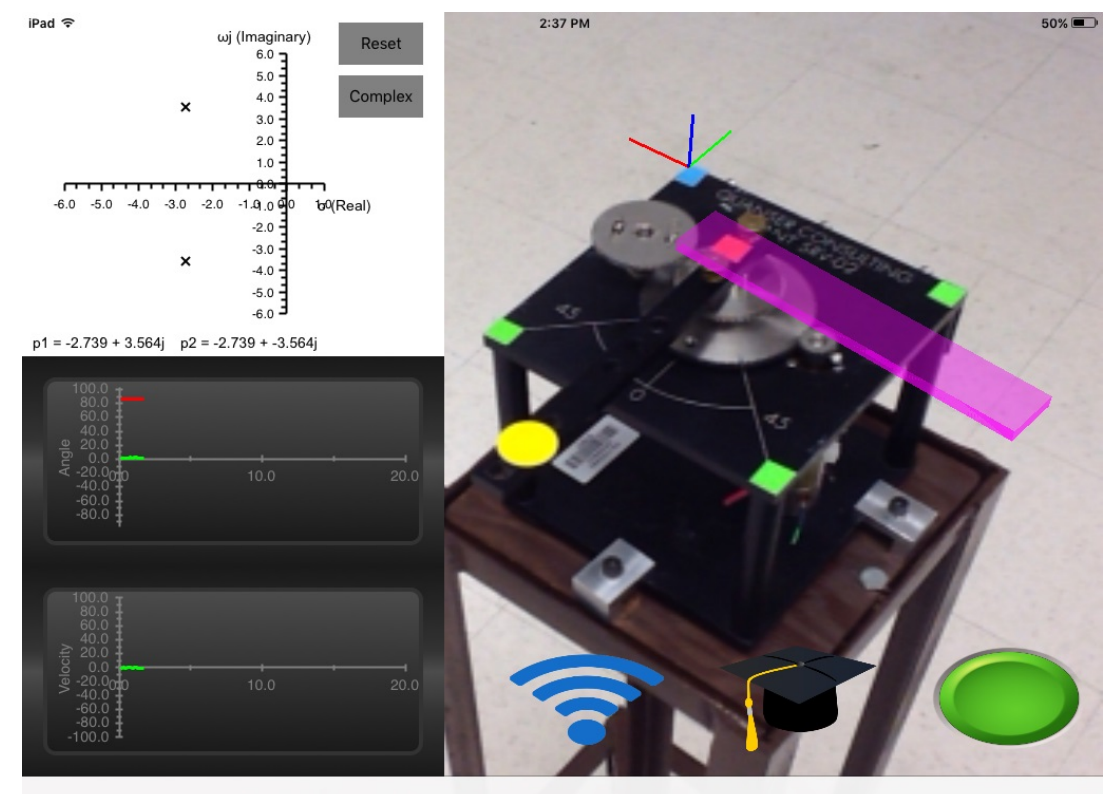

Figure 2: Screenshot of the interface developed for a motor test-bed.

top left-hand view of the interface. By pressing a button that looks like a Wi-Fi symbol, the application is switched to the connect mode wherein a window allows the learner to test and troubleshoot her Wi-Fi connection with the test-bed. By pressing the picture of the graduation cap, the application is switched to the learn mode, which provides a brief walk through the theory behind the content to be explored in the design mode, including an introduction to the responses of systems exhibiting a range of damping and stability conditions and the explanation of the poles of a second-order linear system (see Figure 3).

The design mode, which is the default mode of the interface, is accessed by pressing again the button corresponding to the current mode of the interface. In the design mode, an interactive pole-zero plot allows learners to tap at any desired location to place the closed-loop poles of the system. In the top right corner of the plot, a buttons allows learners to reset the plot. Another button allows learners to switch between two modes of pole selection. In the first mode, only the $x$ coordinates of tapped locations are used to place poles on the real axis of the plot. In the second mode, the $x$ - and $y$-coordinates are used to render a complex pole at the tapped location and another pole at the corresponding complex-conjugate location. The closed-loop poles selected by the learner are used to redesign the controller to drive the test-bed. The result is an interface that allows learners to 


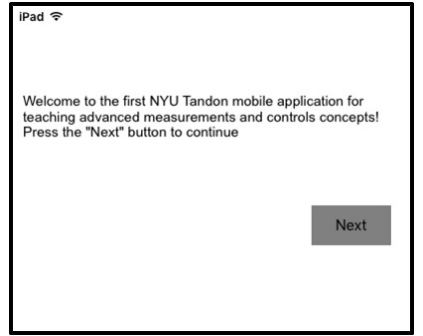

(a)

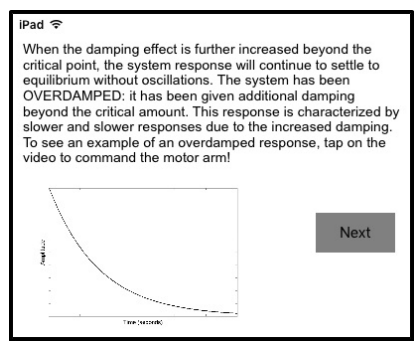

(d)

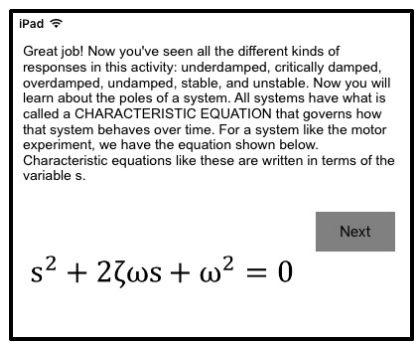

(g)

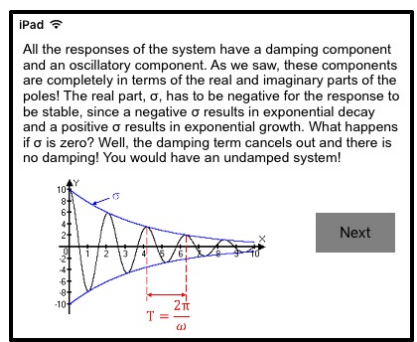

(j)

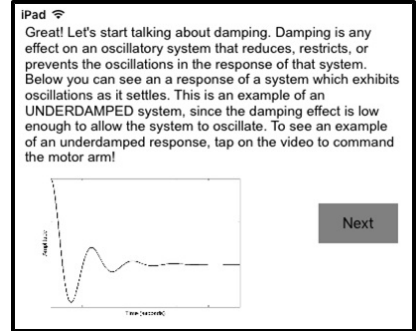

(b)

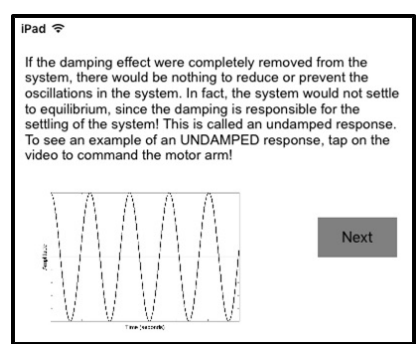

(e)

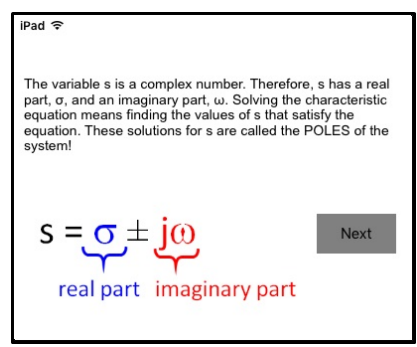

(h)

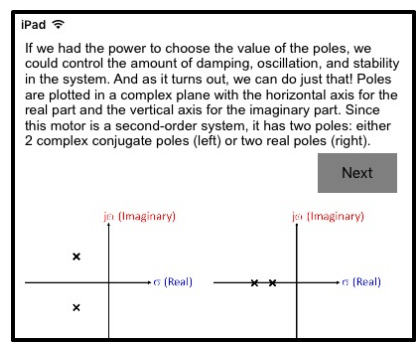

(k)

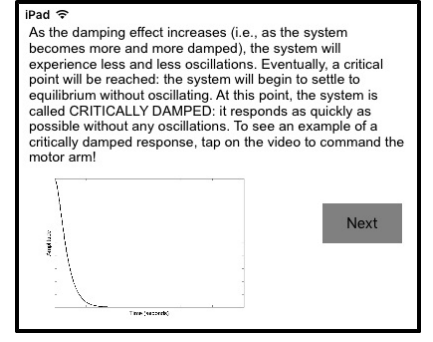

(c)

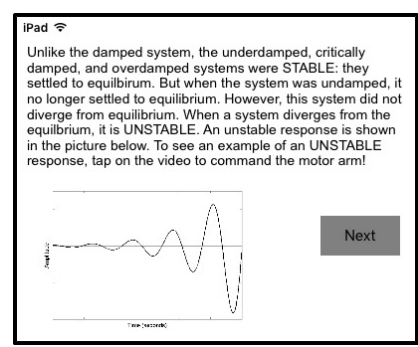

(f)

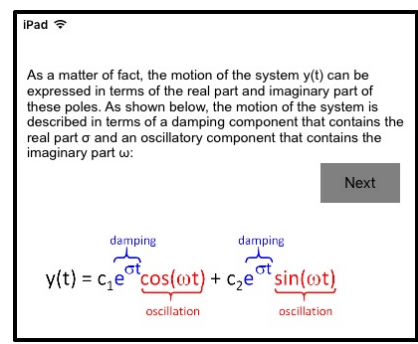

(i)

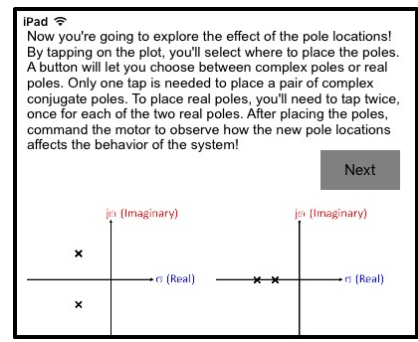

(1)

Figure 3: Screenshots of the educational material provided in the learn mode of the interface. 
interactively alter the system's behavior on the fly.

The bottom left-hand view of the interface displays in real time plots of the angular position and velocity of the motor arm as measured by the mobile device. Together with the other two views, these plots enable learners to make important connections between visually observed responses of the motor arm, the corresponding response plots of measured variables, and the associated values of parameters so that learners may understand the effects that parameters have on system behavior and the presence of certain phenomena (e.g., overshoot, oscillations, and steady-state error).

\subsection{Evaluation Design ${ }^{2.1}$}

To evaluate the educational effectiveness and user experience interacting with the proposed system, a study was conducted with 75 undergraduate students in the mechanical and aerospace engineering department. The students were introduced to the study and offered the option to participate in an interactive educational activity near the end of the Fall 2015 semester. Of these 75 participants, 50 had just completed a laboratory course in Measurement Systems and the other 25 had just completed a laboratory course in Automatic Control, a course that the students take following the Measurement Systems course. Thus, the participants from the Automatic Control course participated in the study after already having been exposed to the topics covered in the study using traditional classroom and hands-on laboratory techniques. To assess the educational effectiveness and user experience associated with using the proposed system, the activity consisted of three parts. First, before performing the interactive activity, participants anonymously answered a pre-assessment (see Appendix A) containing three ungraded questions and 20 graded questions, which were used to assess their level of familiarity and knowledge in the topic areas, respectively. Figure 4 shows the participants' self-reported level of familiarity with each of the topic areas according to the following 5-point scale: the participant has (0) never heard of the topic, (1) heard of the topic, (2) taken a course that discussed the topic, (3) knows a decent amount about the topic, (4) knows the topic very well. Next, the participants were handed the tablet device, were shown the mobile application, and given a 1-minute introduction to the interface and the interactive activity to be performed. The participants were then asked to complete the interactive activity, which required an average of 10 minutes per person. Finally, the participants anonymously responded to a post-assessment (see Appendix B), which contained an ungraded survey used to evaluate the respondents' perception of their experience with the system, and 


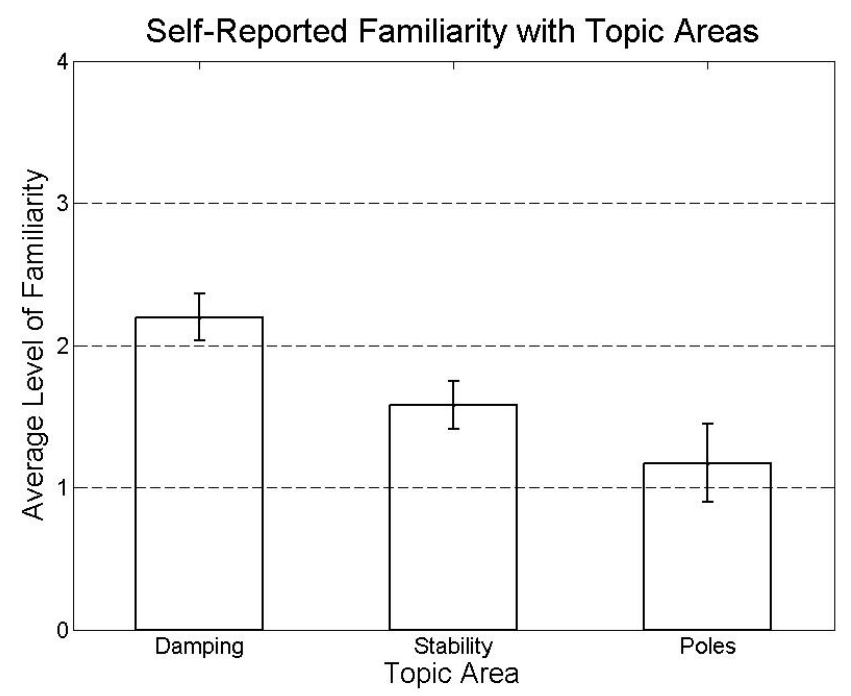

Figure 4: The average levels of familiarity reported by participants in each topic area.

the same 20 graded questions to determine whether the respondents demonstrate any significant knowledge gain in the topic areas.

\section{Resultts ${ }^{2.1}$}

\subsection{Assessment Results}

To investigate the educational effectiveness of the proposed system, four scores are assigned to each of the participants' pre- and post-assessments, one for each of the three topic areas being tested (i.e., system damping, stability, and poles), and one overall score. Each assessment item was classified as belonging to one or more of the three topic areas. Specifically, damping, stability, poles were included in 8, 5, and 15 assessment items, respectively. Table 1 shows the average percentage of points obtained by the 75 participants in each topic area in preand post-assessment. Table 1 also shows the average percentage improvement of participants from pre- to post-assessment. Paired one-tailed $t$-tests were used to determine whether or not the improvements were statistically significant.

As evidenced from Figure 4, participants report higher familiarity with the damping topic than with the stability and pole topics. Yet, as seen from Table 1, participants score highest in the topic of stability. Moreover, the results of two-tailed $t$-tests indicate that participants' pre-assessment scores in the topic of stability are significantly higher than in the topic of poles. These results can be 
Table 1: Scores received by student participants on overall assessment as well as on particular topic areas.

\begin{tabular}{ccccc}
\hline Topic & Pre Score & Post Score & Improvement & Statistically Significant? \\
\hline Damping & $48.24 \%$ & $66.90 \%$ & $38.69 \%$ & YES $\left(p=4.76 \times 10^{-6}<0.05\right)$ \\
\hline Stability & $52.11 \%$ & $85.07 \%$ & $63.24 \%$ & YES $\left(p=1.76 \times 10^{-14}<0.05\right)$ \\
\hline Poles & $41.85 \%$ & $58.65 \%$ & $40.14 \%$ & YES $\left(p=1.67 \times 10^{-7}<0.05\right)$ \\
\hline Overall & $46.13 \%$ & $63.45 \%$ & $37.56 \%$ & YES $\left(p=2.94 \times 10^{-10}<0.05\right)$ \\
\hline
\end{tabular}

Table 2: Scores received by students taking Measurement Systems (MS) course compared to those taking Automatic Control (AC) course.

\begin{tabular}{ccccc}
\hline Metric & MS Average & AC Average & Difference (AC-MS) & Statistically Significant? \\
\hline Damping (Pre) & $56.00 \%$ & $29.76 \%$ & $-26.24 \%$ & YES $(p=0.0012<0.05)$ \\
\hline Damping (Post) & $70.50 \%$ & $58.33 \%$ & $-12.17 \%$ & NO $(p=0.1805>0.05)$ \\
\hline Damping (Improvement) & $25.89 \%$ & $96.00 \%$ & $70.11 \%$ & YES $(p=0.0238<0.05)$ \\
\hline Stability (Pre) & $50.80 \%$ & $55.24 \%$ & $4.44 \%$ & NO $(p=0.5365>0.05)$ \\
\hline Stability (Post) & $86.80 \%$ & $80.95 \%$ & $-5.85 \%$ & NO $(p=0.2477>0.05)$ \\
\hline Stability (Improvement) & $70.87 \%$ & $46.54 \%$ & $-24.33 \%$ & NO $(p=0.2445>0.05)$ \\
\hline Poles (Pre) & $39.43 \%$ & $47.62 \%$ & $8.19 \%$ & NO $(p=0.1722>0.05)$ \\
\hline Poles (Post) & $57.00 \%$ & $62.59 \%$ & $5.59 \%$ & NO $(p=0.3514>0.05)$ \\
\hline Poles (Improvement) & $44.56 \%$ & $31.44 \%$ & $-13.12 \%$ & NO $(p=0.7110>0.05)$ \\
\hline Overall (Pre) & $46.10 \%$ & $46.19 \%$ & $0.09 \%$ & NO $(p=0.9856>0.05)$ \\
\hline Overall (Post) & $63.00 \%$ & $64.52 \%$ & $1.52 \%$ & NO $(p=0.7702>0.05)$ \\
\hline Overall (Improvement) & $36.66 \%$ & $39.68 \%$ & $3.02 \%$ & NO $(p=0.2329>0.05)$ \\
\hline
\end{tabular}

explained by the fact that participants are exposed to the topic of stability in both the Measurement Systems and Automatic Control courses. In contrast, the topics of damping and poles are covered primarily in the Measurement Systems and Automatic Control courses, respectively. Thus, on the pre-assessment, stability questions proved to be easier for the students (as evidenced in the item analysis discussed below). Most importantly, participants' post-assessment scores demonstrate statistically significant improvements over their pre-assessment scores, both overall as well as in each of the three topic areas.

Table 2 shows the scores from the pre- and post-assessments separated by course taken by the students, as well as the results of two-tailed independent $t$-tests (Sauro \& Lewis (2012)) comparing the pre-assessment scores, post-assessment scores, and improvement in scores achieved by students in each course. Results of pre-assessment scores confirm the observation that students of the Measurement Systems course performed significantly better than those in the Automatic Control course on damping-related questions, and students of the Automatic Control course had a higher average score than those in the Measurement Systems course on the topic of poles. However, on the topic of stability, the two classes had a mean 
difference of less than 5 points. Note that, from pre- to post-assessment, while the Automatic Control students nearly doubled their average score on the dampingrelated questions, the Measurement Systems students achieved a $44.56 \%$ mean improvement on the scores for the topic of poles. The two classes of students performed almost the same overall on their pre- and post-assessments, achieving almost the same improvement of nearly 18 points. These results indicate that experiences with the developed MRLE benefited both classes of students, allowing the participants to refresh the previously learned material, and successfully grasp topics that they had not yet been taught in class.

Finally, we contrast student participants exposed to the content using traditional classroom and hands-on laboratory techniques versus student participants exposed to the content using the MRLE platform. To do so, we contrast the pre-assessment scores of the Automatic Control students (who participated in the study after having been exposed to the content, especially that of poles, using the traditional classroom and hands-on laboratory) versus the post-assessment scores of the Measurement Systems students (who had not previously learned the topic of poles). The results of two-tailed independent $t$-tests indicate that the postassessment scores of Measurement Systems students are significantly higher than the pre-assessment scores of Automatic Control students, both overall $(t(73)=$ $2.8199, p=0.0064<0.05)$ and in the topics of damping $(t(73)=5.2563, p=$ $\left.1.75 \times 10^{-6}<0.05\right)$, stability $\left(t(73)=5.6465, p=3.89 \times 10^{-7}<0.05\right)$, and poles $\left(t(73)=7.0181, p=1.61 \times 10^{-9}<0.05\right) .^{2.2}$

\subsection{Item Analysis}

To validate the assessments used in evaluating the developed MRLE, an analysis is performed wherein each item of the assessments (except for items 5a-5e, whose results are consolidated into that of one five-point item) is examined in terms of its level of difficulty, its ability to discriminate between low and high scorers, and its concentration of distractors. These metrics provide further insights into the performance of the participants on the assessments and on the extent to which they learned using the developed MRLE.

To obtain a measure of the level of difficulty for each item in the assessments, a difficulty index (Reynolds, Livingston, \& Willson, 2008) is computed as the percentage of participants who answered the item correctly. Counter-intuitively, a larger value of difficulty index for an item indicates that more participants answered it correctly and thus the item is easier. Table 3 shows the difficulty indices calculated for each item for both the pre-assessment and post-assessment. This is

useful data for comparing the difficulty of test items within each assessment and to 
Table 3: Difficulty index for each assessment item.

\begin{tabular}{ccccc}
\hline Item & Topic (D,S,P) & Difficulty Index (Pre) & Difficulty Index (Post) & Difficulty Differential \\
\hline $1 \mathrm{a}$ & $\mathrm{D}$ & $29.58 \%$ & $57.75 \%$ & $95.24 \%$ \\
\hline $1 \mathrm{~b}$ & $\mathrm{D}$ & $25.35 \%$ & $53.52 \%$ & $111.11 \%$ \\
\hline $1 \mathrm{c}$ & $\mathrm{D}$ & $80.28 \%$ & $90.14 \%$ & $12.28 \%$ \\
\hline $1 \mathrm{~d}$ & $\mathrm{D}$ & $57.75 \%$ & $66.20 \%$ & $14.63 \%$ \\
\hline $2 \mathrm{a}$ & $\mathrm{S}$ & $85.92 \%$ & $97.18 \%$ & $13.11 \%$ \\
\hline $2 \mathrm{~b}$ & $\mathrm{~S}$ & $57.75 \%$ & $83.10 \%$ & $43.90 \%$ \\
\hline $3 \mathrm{a}$ & $\mathrm{DP}$ & $57.75 \%$ & $63.38 \%$ & $9.76 \%$ \\
\hline $3 \mathrm{~b}$ & $\mathrm{DP}$ & $57.75 \%$ & $52.11 \%$ & $-9.76 \%$ \\
\hline $3 \mathrm{c}$ & $\mathrm{DP}$ & $45.07 \%$ & $43.66 \%$ & $-3.13 \%$ \\
\hline $3 \mathrm{~d}$ & $\mathrm{DP}$ & $33.80 \%$ & $42.25 \%$ & $25.00 \%$ \\
\hline $4 \mathrm{a}$ & $\mathrm{SP}$ & $33.80 \%$ & $88.73 \%$ & $162.50 \%$ \\
\hline $4 \mathrm{~b}$ & $\mathrm{SP}$ & $29.58 \%$ & $70.42 \%$ & $138.10 \%$ \\
\hline 5 & $\mathrm{P}$ & $40.00 \%$ & $57.75 \%$ & $14.37 \%$ \\
\hline 6 & $\mathrm{P}$ & $29.58 \%$ & $33.80 \%$ & $15.29 \%$ \\
\hline 7 & $\mathrm{P}$ & $45.07 \%$ & $52.11 \%$ & $60.53 \%$ \\
\hline 8 & $\mathrm{SP}$ & $53.52 \%$ & $85.92 \%$ &
\end{tabular}

compare items by their difficulty differential, or the percentage change in difficulty from the pre- to post-assessment. One interesting observation from Table 3 is that the questions related to stability were among the items with the largest difficulty index in the pre-assessment and the questions on the relationship between the pole locations and the stability of the system had the largest pre- to post-assessment difficulty differential. These results explain the significantly higher stability scores obtained in the pre-assessment and the large improvements in stability scores obtained by participants from pre-assessment to post-assessment compared to the other topic areas. Furthermore, the difficulty differential of all but items $3 b$ and $3 c$ were positive, indicating that participants' performance improved for each item except for these two items. Thus, in future work, we will consider a redesign of the MRLE to ensure that the concepts assessed in the two items with negative differential, as well as the items with low post difficulty index $(3 \mathrm{~d}, 6)$, are reinforced in the material presented and activity performed. An item's discrimination index (Reynolds et al., 2008) is a measure of the extent to which the item can distinguish between more and less knowledgeable participants, based on a correlation between their performance on the item and their overall performance on the assessment. For an item with high discrimination, participants who respond to the item correctly also do well on the overall assessment while participants who respond to the item incorrectly tend to do poorly on the overall assessment. In other words, the discrimination index can be used as a measure of the validity of a test item, such that items with high discrimination index are those for which 
Table 4: Discrimination index for each assessment item.

\begin{tabular}{ccc}
\hline Item & Discrimination Index (Pre) & Discrimination Index (Post) \\
\hline $1 \mathrm{a}$ & 0.230 & 0.434 \\
\hline $1 \mathrm{~b}$ & 0.153 & 0.343 \\
\hline $1 \mathrm{c}$ & 0.331 & 0.277 \\
\hline $1 \mathrm{~d}$ & 0.191 & 0.385 \\
\hline $2 \mathrm{a}$ & 0.210 & -0.009 \\
\hline $2 \mathrm{~b}$ & 0.152 & 0.212 \\
\hline $3 \mathrm{a}$ & 0.230 & 0.525 \\
\hline $3 \mathrm{~b}$ & 0.222 & 0.325 \\
\hline $3 \mathrm{c}$ & 0.313 & 0.318 \\
\hline $3 \mathrm{~d}$ & 0.383 & 0.397 \\
\hline $4 \mathrm{a}$ & 0.435 & 0.249 \\
\hline $4 \mathrm{~b}$ & 0.368 & 0.398 \\
\hline 5 & 0.392 & 0.501 \\
\hline 6 & 0.264 & 0.128 \\
\hline 7 & 0.297 & 0.272 \\
\hline 8 & 0.326 & 0.185 \\
\hline
\end{tabular}

participants are marked correct or incorrect based on their level of knowledge or ability rather than something else such as chance or test bias. Table 4 shows the discrimination index calculated for each item on the pre- and post-assessments using a corrected item-total correlation. Although the possible range of the discrimination index is -1.0 to 1.0 , note that there is an interaction between an item's difficulty index and it's discrimination index. For example, items that are either very easy or very difficult will not be very discriminating. Thus, items with good discrimination will typically range above 0.2 . If an item has a discrimination below 0.0, it suggests a problem, as this item is being answered incorrectly by the top performing participants and answered correctly by the bottom performing participants. Using these guidelines, we see that most of the assessment items yield acceptable discrimination index. The result is that the majority of the assessment items appropriately indicate the educational impact of the developed MRLE, with the exception of items $1 \mathrm{~b}, 1 \mathrm{~d}$, and $2 \mathrm{~b}$ in the pre-assessment and items $2 \mathrm{a}, 6$, and 8 in the post-assessment, which have either marginal or low discrimination levels and will need to be revised for experiments with future cohorts.

\subsection{User Experience Results}

To assess aspects of the user experience associated with the MRLE, such as usability, learnability, and engagement, participants were asked to respond to a questionnaire inspired from the Post-study System Usability Questionnaire (Lewis, 2002). Figure 5 shows the participants' level of agreement with the following 12 


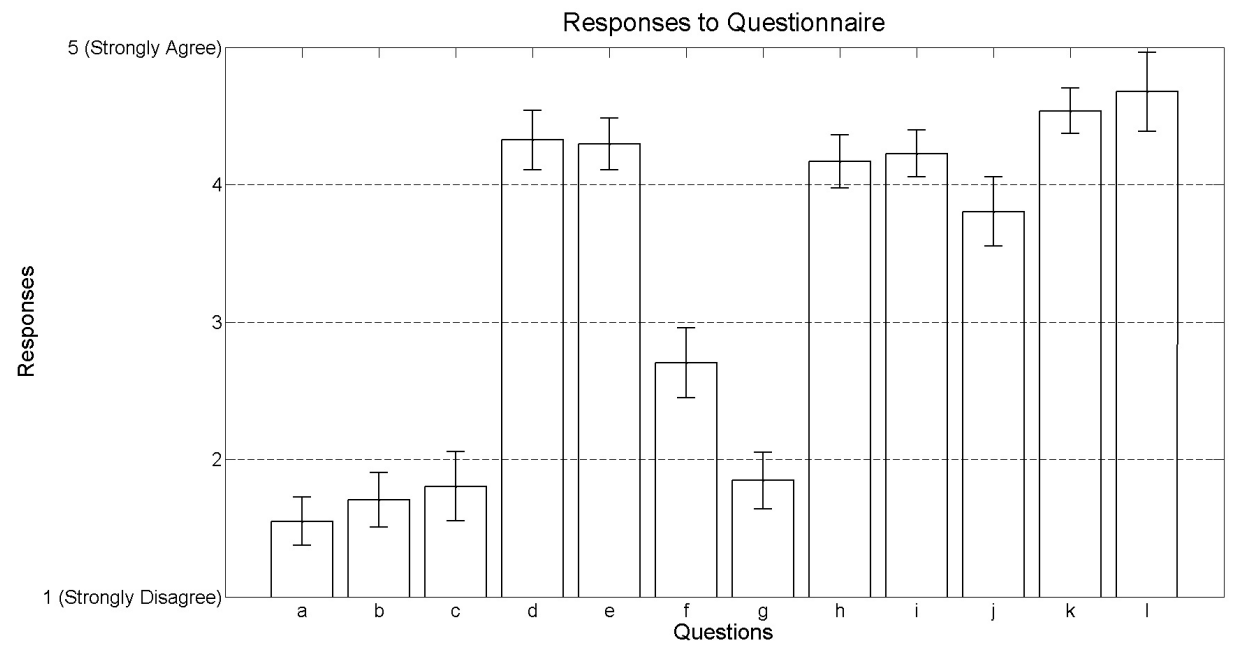

Figure 5: Participant responses to the user experience questionnaire.

statements on a 5-point scale (1: strong disagreement and 5: strong agreement):

(a) It was difficult to navigate the app.

(b) It was difficult to work with the interactive plots in the app.

(c) It was difficult to command the test-bed using the touchscreen.

(d) It was fun interacting with the augmented reality graphics.

(e) Overall, the app made it easy and fun to interact with the motor test-bed.

(f) I required assistance from the TA in order to use the app.

(g) It took a long time for me to become comfortable using the app.

(h) I have improved my understanding of damping using this app.

(i) I have improved my understanding of stability using this app.

(j) I have improved my understanding of closed-loop poles using this app.

(k) Overall, I would recommend this app to students for learning about damping, stability, and closed-loop poles.

(l) I would like to see apps like this introduced into the engineering lab curriculum.

In terms of difficulty, participants are largely in disagreement that the interface is difficult to navigate and to use for interacting with the test-bed. In fact, participants report having a fun and easy experience with the MRLE. Although they required some assistance from a teaching assistant (TA) to use the application, the participants report becoming comfortable with the interface after only a short time. 
Note that although the participants report having improved their understanding of the concepts taught by the developed MRLE, their level of agreement is slightly lower for the poles concepts as compared to damping and stability concepts. This result is expected, since previously presented content assessment results indicated that the poles topic was more difficult than the other two topics, even though participants achieved a $40.14 \%$ improvement in their scores related to this topic. Finally, participant responses to the questionnaire point to overall favorable experiences using the MRLE, with the majority reporting in strong agreement that they would recommend the interface for learning and that they have a desire to see such interfaces introduced into the curriculum.

\subsection{Participant Comments and Recommendations}

Of the 75 participants in the study, 52 provided descriptive comments and suggestions that can be considered for future improvements to the interaction experience with the MRLE. Comments included praises, which are characterized as either positive comments that offered no recommendation for improvement or suggestions that were positively biased, such as

"[This is] exactly the kind of learning tool I've wanted for a long time" - Participant \# 34.

Also encountered were criticisms, which are defined as either negative comments that offered no recommendation for improvement or suggestions that were negatively biased, such as

"It was a little difficult to use" - Participant \# 45.

Finally, participants also left suggestions, which are distinguished as comments that provided recommendations for improvement without being particularly positive or negative in nature, such as

"It would be great if there were an automated voice reading the content or a video presentation of the content" - Participant \# 12.

Figure 6 summarizes the percentage of participants who left each type of comment. The results indicate that $31 \%$ of participants left no comments and of the remaining $69 \%$ of participants, $73.5 \%$ left praises, $36.7 \%$ left criticisms, and $59.2 \%$ left suggestions, which correspond to $50.1 \%, 25.4 \%$, and $40.8 \%$ of the total number of participants, respectively. These results are promising because they indicate that a majority of the participants felt affected enough by the activity to 


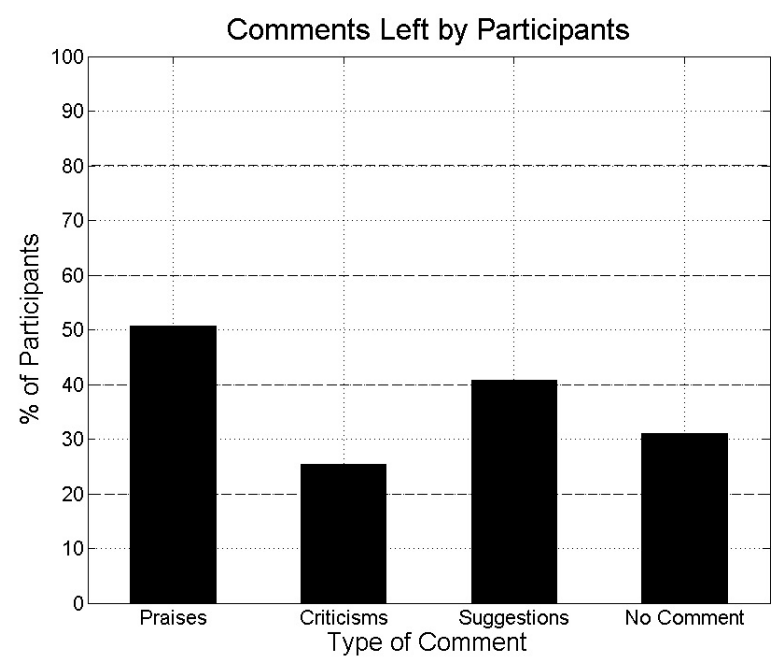

Figure 6: Percentage of participants who left each type of comment in the questionnaire.

leave meaningful comments for the developers, and that a significant portion of the participants were satisfied enough to praise their experience in writing. Praises were taken as validation of the our arguments for integrating systems such as the one proposed in this paper into the laboratory curricula. Many of the praises touched on the enjoyment associated with the interactive aspects of the experiment, the ability to visualize concepts with the aid of AR, and the notion that the intimate link between the interaction on the interface and the observed behavior of the physical system provided a deeper understanding of the concepts. Criticisms served as a reminder that this class of laboratory test-beds is still in its infancy and much work remains to be done to improve the usability and educational impact of interactive applications that also involve the direct manipulation of physical dynamics. Suggestions left by the participants were mainly centered on usability issues associated with the navigation of the interface and on ways in which the activity can be modified to enhance the teaching and learning potential of the proposed system. These suggestions will be considered in the development and testing of future prototypes before the system is incorporated into the curriculum.

\section{Discussion 2.1}

\subsection{Benefits and Drawbacks of MRLE $E^{2.4}$}

During the development and evaluation of the MRLE platform, a variety of notable benefits and drawbacks of the proposed MRLE approach were observed 
vis-à-vis the conventional hands-on, virtual, and remote laboratories. As an extension of traditional hands-on laboratories, the MRLE approach shares some similarities to hands-on laboratories, such as the need for real laboratory equipment that must be purchased and maintained. Moreover, platforms implementing the MRLE approach require learners to be present near the equipment, imposing restrictions on access that make the MRLE less mobile than virtual and remote laboratories. However, the MRLE approach provides the benefit of real data that cannot be matched by simulation. Furthermore, by requiring that learners set up the equipment used to conduct the experiment, we ensure their exposure to 'unexpected clashes' between theory and practice that foster design skills essential for understanding the role of experiments, but are often missing from simulated and remote labs (Ma \& Nickerson, 2006). ${ }^{2.4}$

Extensive research has investigated differences in the ability of students to achieve learning outcomes, such as improved conceptual understanding, using virtual models of concepts encountered in non-traditional (virtual and remote) laboratories as compared to physical models used in traditional hands-on laboratory format (Corter, Esche, Chassapis, Ma, \& Nickerson, 2011; Ma \& Nickerson, 2006). However, research often shows that exposure to both virtual and physical models results in improved content knowledge than using either one of the models alone (Copolo \& Hounshell, 2006). Moreover, the use of multiple linked representations of scientific phenomena has been shown to effectively account for different learning styles and preferences of individual learners (Wu, Krajcik). Thus, learners using the proposed platforms are given access to multimodal learning experiences through interactions with both physical and virtual representations of phenomena. ${ }^{2.5}$

Laboratory equipment can be expensive, and to dedicate laboratory-grade computing, data acquisition and control, power amplification, sensing, and actuation to each station can be a significant financial burden. Many laboratories will pay for equipment once and then cannot upgrade their facilities for many years. The limited number of available laboratory stations often forces students to work in teams that are too large to support meaningful individual experiences in the laboratory. Unlike virtual and remote laboratories, the MRLE approach economizes in-lab activities without sacrificing the benefits of hands-on experiences. Specifically, the SSCC capabilities of mobile devices enable them to perform the same roles as some of the most expensive components of traditional laboratory stations, allowing students to still work with real equipment while eliminating the cost of redundant laboratory-grade hardware and software. In fact, mobile devices may obviate so much of the traditional laboratory station that new, more portable 
platforms may be feasible using embedded computers to interface with learners' devices. ${ }^{2.4}$

Learners not only already own mobile tools that can be utilized in laboratory activities, they are also already trained and comfortable with using them. Thus, a significant amount of time and effort that learners spend familiarizing themselves with laboratory stations can be saved by exploiting the features on their personal devices with which they are already familiar. Among these features are rich interactive visualizations that aid learners in grasping abstract concepts, providing deeply engaging mixed-reality learning experiences right in the learner's hands without the need to sit at a computer station or remove focus from the learning activity. To provide these experiences, laboratory platforms must leverage a host of the mobile devices' native hardware and software resources, requiring native applications to be developed. A major challenge for laboratories interested in implementing the proposed approach is the effort required to develop interfaces. Different mobile platforms (e.g., Android, iOS, Windows) rely on different operating systems, computer architectures, processor technologies; carry different types of sensors and sensors with different specifications; and offer developers different application programming interfaces and different development environments that require knowledge of different programming languages. Moreover, because the proposed MRLEs employ state-of-the-art features of mobile technologies, some platforms may still not have specifications needed to produce stable solutions. Finally, since different test-beds demonstrate different scientific and engineering principles, their state may not be fully measurable or observable by the mobile device. Thus, as with remote laboratories, different test-beds will be amenable to MRLEs to varying degrees, and developers must assess on a case-by-case basis which sensors, algorithms, and interface techniques may be useful to a particular implementation. . $^{2.4}$

\subsection{Rationale for Identical Pre- and Post-Assessments ${ }^{1.2}$}

Pre- and post-assessments are designed to measure the same learning outcomes at the same level of difficulty using similar methods, producing scores that can be compared to one another to demonstrate any resulting growth. Although one approach is to administer identical pre- and post-assessments, they are often designed to be equivalent without being identical to avoid the risk of introducing a testing effect, in which knowledge is gained through familiarity with the test items rather than the treatment delivered between assessments. However, a testing effect normally occurs when students are provided feedback of their performance on the pre-assessment, either from discussion with the instructor or a fellow student, or 
by checking answers themselves. This feedback was prevented in our design of the experiment by having student participants tested individually, being directed to perform the activity immediately after the pre-assessment and then immediately to complete the post-assessment. Furthermore, if a testing effect had occurred in the assessment phase, it would manifest itself as an increase in the difficulty index from pre- to post-assessment (indicating that the test item became easier to answer) coupled with a corresponding decrease in the discriminating index from pre- to post-assessment (indicating that the item became easier due to some reason other than the activity conducted by student). However, as evidenced in the discussion of the item analysis results (subsection 4.2), all but two of the test items with large increases in difficulty are accompanied by discrimination indices that either increase or remain relatively unchanged. Furthermore, the two items that have decreases in discrimination index continue to have reasonably high values (approximately 0.2 ). Thus, no significant testing effect occurred in the study. ${ }^{1.2}$

Moreover, the use of a post-assessment that was identical to the pre-assessment actually showed students how their understanding improved throughout the course of the activity. As a pre-assessment, the items facilitated an inquiry-based learning process, presenting students with the problems and scenarios that they were expected to explore further in the activity. As a post-assessment, the items allowed students to see how their thinking changed and what they learned since the preassessment. Thus, in this study, the advantages of using identical pre- and postassessments (i.e., allowing for the most direct and meaningful item analysis to be conducted, preparing students expectations for the system behaviors to explore, etc.) outweighed the potential limitations (i.e., the possibility that improvements were partially due to knowledge absorbed while taking the pre-assessment). ${ }^{1.2}$

\subsection{Results $^{2.2}$}

This study has presented the development and evaluation of an educational platform that integrates the functional and user interaction capabilities of learners mobile devices directly with a laboratory test-bed in a manner that has not been studied previously. In evaluating the educational effectiveness of the platform in terms of conceptual understanding, student participants were found to demonstrate significant improvements in their level of content knowledge in several topic areas of dynamic systems and control after using the MRLE platform compared to before using the MRLE platform and compared to student participants exposed to the content using traditional classroom and hands-on laboratory techniques. Thus, the hypothesis that the proposed platform is educationally effective is deemed validated. Moreover, in evaluating the user experience associated with the use of the 
MRLE platform, results indicate that student participants report having generally favorable experiences with the platform in both their responses to questionnaires and in their written comments. Thus, the hypothesis that the proposed platform renders positive student perceptions is deemed validated..$^{2.2}$

\section{Conclusions ${ }^{2.1}$}

Laboratories must constantly adapt their styles and strategies to changes in the economic and technological landscape. In this paper, a novel approach was proposed in which the hardware and software of mobile devices and the physical dynamics of laboratory test-beds are blended to create mobile mixed-reality learning environments. By developing mobile applications that incorporate interactive plots and AR, unique cost-effective and stimulating hands-on educational experiences can be provided via learners' personal devices. Thus, by blending elements and obtaining benefits of both traditional and non-traditional laboratory approaches, the mixed-reality approach proposed in this paper takes a step towards closing the gap between them. ${ }^{2.5}$ To illustrate the proposed MRLE approach, a motor-based platform was developed to teach concepts of dynamic systems and control. Results of an evaluation conducted using the platform with undergraduate engineering students validate the hypotheses that student participants demonstrate significant improvement in content knowledge and report having significantly beneficial experiences after using the MRLE platform compared to before using the MRLE platform and compared to student participants exposed to the content using traditional classroom and hands-on laboratory techniques. ${ }^{2.2}$ Furthermore, the participants left useful comments that have been considered in the improvement of the MRLE. Finally, as the proposed MRLE approach continues to eliminate the expense of laboratory grade-equipment, it may become more technically and economically feasible to develop completely portable platforms. Thus, the learning experiences provided by MRLEs may become more accessible as students would be able to take the portable platforms with them out of the laboratory for performing experiments without time or place restrictions. ${ }^{2.4}$ 


\section{Appendix A. Pre-Assessment}

The pre-assessment begins with three questions asking participants to report their level of understanding of damping in a dynamic system, of the stability of a dynamic system, and of the poles of a dynamic system. Then, the preassessment asks 20 questions related to the topics of damping, stability, and poles. The first 4 questions test the student's familiarity with different damping conditions by displaying pictures of time responses of systems with damping ratios of $\zeta=0,0.25,1$, and 3 , and asking the student to choose the appropriate damping condition associated with each response (Fig. A.1(a)). The next 2 questions test the student's understanding of the difference between unstable and stable responses by presenting pictures of a stable and an unstable response of a system and asking the student to choose the appropriate stability condition associated with each response. The next 6 questions test the student's understanding of the effect of pole locations on the damping and stability of a system. The student is again asked to select the associated damping condition and stability condition, however instead of providing pictures of time responses, pictures of the complex plane with pole locations in different configurations are provided (Fig. A.1(b)). The next 5 questions involve a column of time responses and a column of pole configurations and require the student to match the items from each column (Fig. A.1(c)). This visual question tests whether students can associate the pole locations to the corresponding response of the system, even if the student cannot necessarily identify the responses. The remaining 3 multiple-choice questions test the student's ability to recognize certain behavior trends of the system in response to certain migrations of the poles across the complex plane (Fig. A.1(d)).

\section{Appendix B. Post-Assessment}

The post-assessment begins with the same 20 questions that students had to answer in the pre-assessment testing their understanding of the relationships between the damping and stability of a system's response and the locations of that system's poles (Fig. A.1(a)-A.1(d)). Following the quiz, students are asked to agree or disagree with 12 statements according to a five-point Likert scale (Fig. A.1(d)). At the end of the post-assessment, students were given space where they were encouraged to leave any comments, suggestions, criticisms, or praises that they had regarding their experience with using the proposed system. 

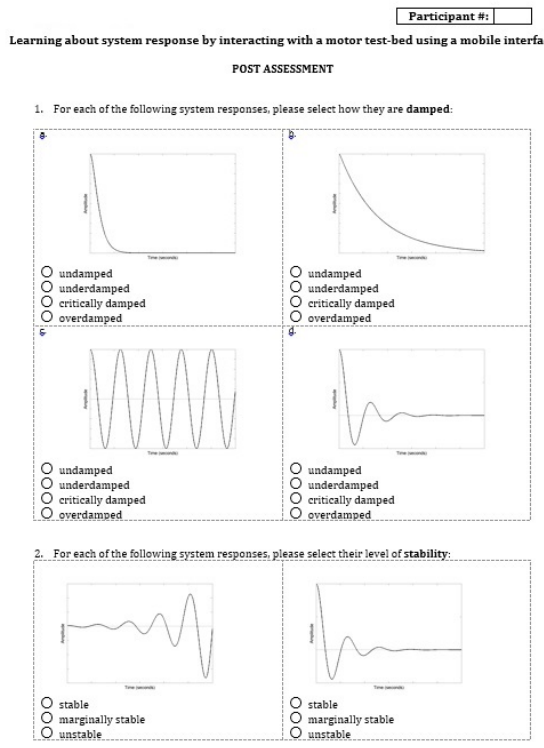

(a)

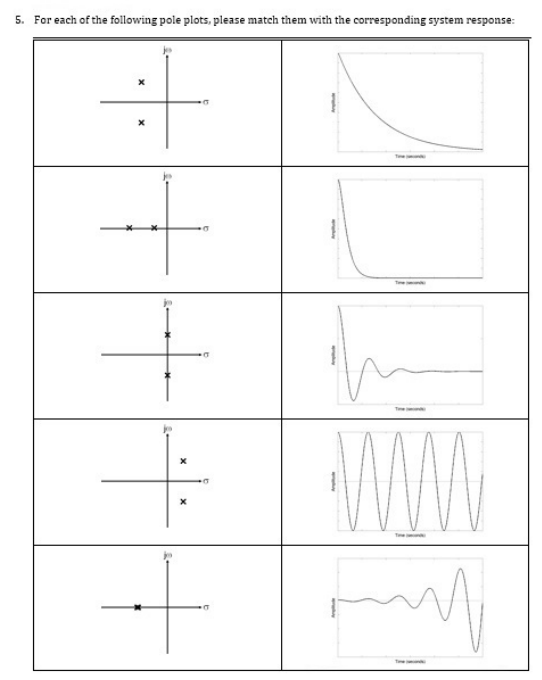

(c)

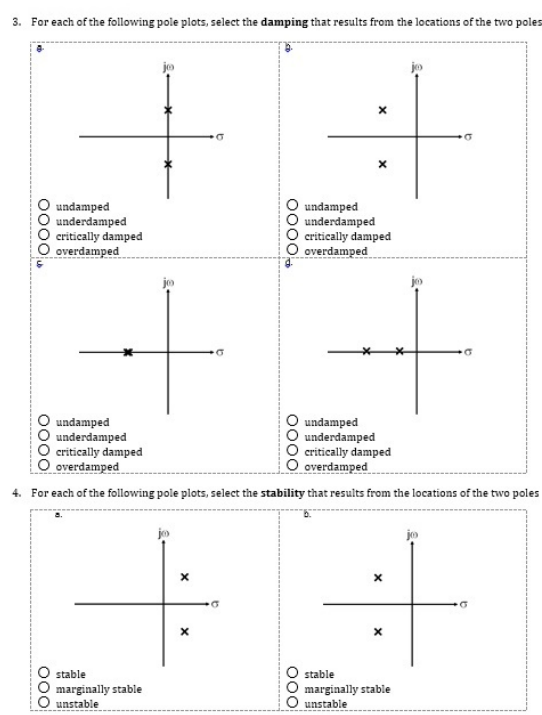

(b)

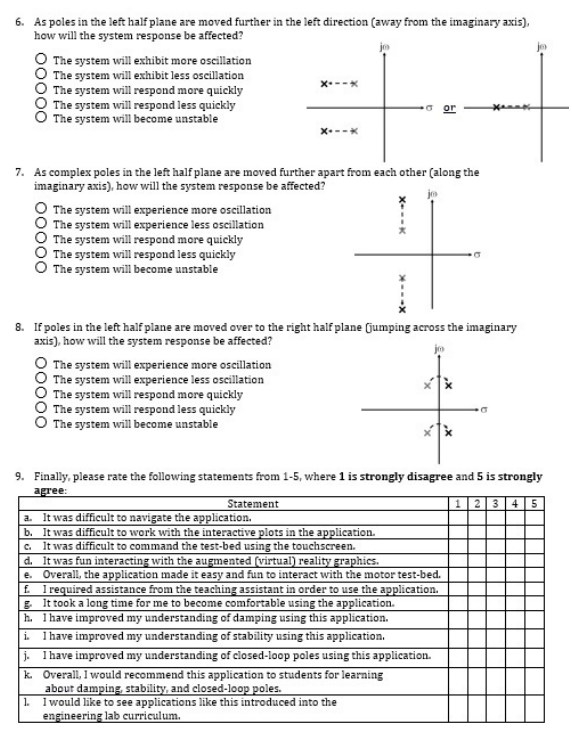

(d)

Figure A.1: The post-assessment issued to undergraduate student participants after interacting with the motor test-bed using the mobile interface. 


\section{References}

Apkarian, J. (1995). A comprehensive and modular laboratory for control systems design and implementation. Toronto, Canada: Quanser Consulting.

Bonnington, C. In less than two years, a smartphone could be your only computer. (2015). Wired. http://www.wired.com/2015/02/ smartphone-only-computer/ Accessed 02.10.2015.

Chang, C.W., Lee, J.H., Wang, C.Y., \& Chen, G.D. (2010). Improving the authentic learning experience by integrating robots into the mixed-reality environment. Computers \& Education, 55(4), 1572-1578.

Copolo, C.E., \& Hounshell, P.B. (1995). Using three-dimensional models to teach molecular structures in high school chemistry. Journal of Science Education and Technology, 4(4), 295-305.

Corter, J.E., Esche, S.K., Chassapis, C., Ma, J., \& Nickerson, J.V. (2011). Process and learning outcomes from remotely-operated, simulated, and hands-on student laboratories. Computers \& Education, 57(3), 2054-2067.

da Silva, J.B., Rochadel, W., Marcelino, R., Gruber, V., \& Bilessimo, S.M.S. (2013). Mobile remote experimentation applied to education. In O. Dziabenko, \& J. García-Zubia (Eds.), IT Innovative practices in secondary schools: Remote experiments (pp. 281-302). Bilbao: University of Deusto.

de Lima, J.P.C., Rochadel, W., Silva, A.M., Simão, J.P.S., da Silva, J.B., \& Alves, J.B.M. (2014). Application of remote experiments in basic education through mobile devices. In IEEE Global Engineering Education Conference, Istanbul, Turkey, 1093-1096.

Feisel, L., \& Rosa, A. (2005). The role of the laboratory in undergraduate engineering education. Journal of Engineering Education, 94(1), 121-130.

Frank, J.A., \& Kapila, V. (2014). Development of mobile interfaces to interact with automatic control experiments [Focus on education]. IEEE Control Systems, 34(5), 78-98.

Frank, J.A., \& Kapila, V. (2016). Towards teleoperation-based interactive learning of robot kinematics using a mobile augmented reality interface on a tablet. In Indian Control Conference, Hyderabad, India, 385-392. 
Frank, J.A., Brill, A., \& Kapila, V. (2016) Interactive mobile interface with augmented reality for learning digital control concepts. In Indian Control Conference, Hyderabad, India, 85-92.

Frank, J.A., Gómez, J.A.D., \& Kapila, V. (2015). Using tablets in the vision-based control of a ball and beam test-bed. In Proceedings of the International Conference on Informatics in Control, Automation and Robotics, Colmar, Alsace, France, 92-102.

Gillett, F. Why tablets will become our primary computing device. (2012). Forrester. http://blogs.forrester.com/frank_gillett/ 12-04-23-why_tablets_will_become_our_primary_ computing_device/ Accessed 08.27.2016.

Haralick, R.M., Joo, H., Lee, C.N., Zhuang, X., Vaidya, V.G., \& Kim, M.B. (1989). Pose estimation from corresponding point data. IEEE Transactions on Systems, Man, and Cybernetics, 19(6), 1426-1446.

Hartley, R., \& Zisserman, A. (2003). Multiple View Geometry in Computer Vision. New York: Cambridge University Press.

Hesser, T., \& Schwartz, P. (2013). iPads in the science laboratory: Experience in designing and implementing a paperless chemistry laboratory course. Journal of STEM Education: Innovations \& Research, 14(2), 5-9.

Kaufmann, H., \& Schmalstieg, D. (2003). Mathematics and geometry education with collaborative augmented reality. Computers \& Graphics, 27(3), 339-345.

Kerawalla, L., Luckin, R., Seljeflot, S., \& Woolard, A. (2006). Making it real: Exploring the potential of augmented reality for teaching primary school science. Virtual Reality, 10(3-4), 163-174.

Klein, P., Gröber, S., Kuhn, J., \& Müller, A. (2014). Video analysis of projectile motion using tablet computers as experimental tools. Physics Education, 49(1), $37-40$.

Klein, P., Hirth, M., Gröber, S., Kuhn, J., \& Müller, A. (2014). Classical experiments revisited: Smartphones and tablet PCs as experimental tools in acoustics and optics. Physics Education, 49(4), 412. 
Kuhn, J., \& Vogt, P. (2013). Applications and examples of experiments with mobile phones and smartphones in physics lessons. Frontiers in Sensors, 1(4), 6773.

Kuhn, J., Molz, A., Gröber, S., \& Frübis, J. (2014). iRadioactivity: Possibilities and limitations for using smartphones and tablet PCs as radioactive counters. The Physics Teacher, 52(6), 351-356.

Lane, N.D., Miluzzo, E., Lu, H., Peebles, D., Choudhury, T., \& Campbell, A.T. (2010). A survey of mobile phone sensing. IEEE Communications Magazine, $48(9), 140-150$.

Lewis, J. R. (2002). Psychometric evaluation of the PSSUQ using data from five years of usability studies. International Journal of Human-Computer Interaction, 14(3-4), 463-488.

Liarokapis, F., Mourkoussis, N., White, M., Darcy, J., Sifniotis, M., Petridis, P., et al. (2004). Web3d and augmented reality to support engineering education. World Transactions on Engineering and Technology Education, 3(1), 11-14.

Liu, J., Hu, S., Thiagarajan, J.J., Zhang, X., Ranganath, S., Banavar, M. K., et al. (2012). Interactive DSP laboratories on mobile phones and tablets. In IEEE International Conference on Acoustics, Speech and Signal Processing, Kyoto, Japan, 2761-2764.

Ma, J., \& Nickerson, J.V. (2006). Hands-on, simulated, and remote laboratories: A comparative literature review. ACM Computing Surveys, 38(3), 7, 1-24.

Maier, P., Tönnis, M., \& Klinker, G. (2009). Augmented reality for teaching spatial relations. In Conference of the International Journal of Arts \& Sciences, Toronto, Canada.

Maiti, A., \& Tripathy, B. (2012). Different platforms for remote laboratories in mobile devices. International Journal of Modern Education and Computer Science, 4(5), 38-45.

Martín-Gutíerrez, J., Contero, M., Alcañiz, M. (2010). Evaluating the usability of an augmented reality based educational application. In Intelligent Tutoring Systems, 296-306. 
May, D., Terkowsky, C., Haertel, T., \& Pleul, C. (2012). Using e-portfolios to support experiential learning and open the use of tele-operated laboratories for mobile devices. In International Conference on Remote Engineering and Virtual Instrumentation, Bilbao, Spain.

Nguyen, L., Barton, S., \& Nguyen, L. (2015). iPads in higher education: Hype and hope. British Journal of Educational Technology, 46(1), 190-203.

Orduña, P., García-Zubia, J., Irurzun, J., López-de-Ipiña, D., \& Rodriguez-Gil, L. (2011). Enabling mobile access to remote laboratories. In Global Engineering Education Conference, Amman, Jordan, 312-318.

Ranganath, S., Thiagarajan, J.J., Ramamurthy, K.N., Hu, S., Banavar, M., \& Spanias, A. (2012). Work in progress: Performing signal analysis laboratories using Android devices. In Frontiers in Education Conference, Seattle, Washington, 1-2.

Reynolds, C.R., Livingston, R.B., \& Willson, V. (2008). Measurement and assessment in education. (2nd ed.). Pearson.

Sauro, J., \& Lewis, J.R. (2012). Quantifying the user experience: Practical statistics for user research. Morgan Kaufmann.

Tian, K., Endo, M., Urata, M., Mouri, K., \& Yasuda, T. (2014). Lunar observation support system using smartphone AR for astronomy education. International Journal of Interactive Mobile Technologies, 8(1), 32-39.

Venkataraman, B. (2009). Visualization and interactivity in the teaching of chemistry to science and non-science students. Chemistry Education Research and Practice, 10(1), 62-69.

Vogt, P., \& Kuhn, J. (2013). Analyzing radial acceleration with a smartphone acceleration sensor. The Physics Teacher, 51(3), 182-183.

Williams, A., \& Pence, H. (2011). Smart phones, a powerful tool in the chemistry classroom. Journal of Chemical Education, 88(6), 683-686.

Wu, H.-K., Krajcik, J.S., \& Soloway, E. (2001). Promoting understanding of chemical representations: Students' use of a visualization tool in the classroom. Journal of Research in Science Teaching, 38(7), 821-842. 


\section{List of changes}

Added (1.1): Even as mobile devi .. . . . . . . . . . . . . . 2

Added (1.1): relieve the need for .. . . . . . . . . . . . 2

Added (2.2): We hypothesize that.. . . . . . . . . . . . . . 2

Added (1.1): The benefits and dra .. . . . . . . . . . . . . 2

Added (1.1): laboratories constant... . . . . . . . . . . . . 3

Added (1.1): However, the .. _. . . . . . . . . . . 3

Added (1.1): The novelty of the w . . . . . . . . . . . . . 4

Added (2.2): To validate the propo .. . . . . . . . . . . . . 5

Added (2.1): In Section 2, we pres . . . . . . . . . . . . . . 5

Added (2.1): Background . . . . . . . . . . . 5

Added (2.4): However, since curre . . . . . . . . . . . . . . . 7

Added (2.5): Thus, an approach th . . . . . . . . . . . . . . 7

Added (2.1): Methodology _. . . . . . . . . . . . 7

Added (2.1): Implementation $\quad \ldots \ldots . \ldots . \ldots$

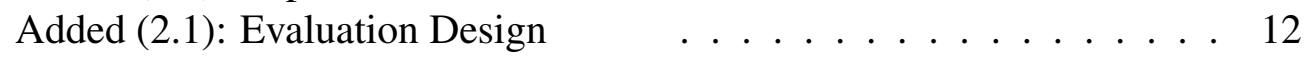

Added (2.1): Results _. . . . . . . . . . . . . 13

Added (2.2): Finally, we contrast s . . . . . . . . . . . . . . . . . . . . . . . . .

Added (2.1): Discussion _............... 20

Added (2.4): Benefits and Drawba .. . . . . . . . . . . . . . 20

Added (2.4): During the developm .. . . . . . . . . . . . . . . . 21

Added (2.5): Extensive research h.. . . . . . . . . . . . . . . 21

Added (2.4): Laboratory equipme... . . . . . . . . . . . . . . . . . . . 22

Added (2.4): Learners not only alr . . . . . . . . . . . . . . . . . . . . . . . . . . . . 22

Added (1.2): Rationale for Identic . . . . . . . . . . . . . . . . . . . . . . 22

Added (1.2): Pre- and post-.. . . . . . . . . . . . . 23

Added (1.2): Moreover, the use of . . . . . . . . . . . . . . . . 23

Added (2.2): Results _. . . . . . . . . . . . . 23

Added (2.2): This study has prese . . . . . . . . . . . . . . . . . . . . . . . . 24

Added (2.1): Conclusions … . . . . . . . . . 24

Added (2.5): Thus, by blending el. . . . . . . . . . . . . . . . . . 24

Added (2.2): validate the hypothes .. . . . . . . . . . . . . . . . . . . . . . . . . . . . 24

Added (2.4): Finally, as the propo... . . . . . . . . . . . . . . 24 